\title{
Phase-Noise Reduction in Self-Injection Locked Oscillators Using Slow-Wave Structures
}

\author{
Mabel Pontón ${ }^{1}$, Franco Ramírez ${ }^{2}$, Amparo Herrera $^{3}$, Almudena Suárez ${ }^{4}$ \\ University of Cantabria, Spain \\ 1' mabel.ponton@unican.es, ${ }^{2}$ ramirezf@unican.es, ${ }^{3}$ herreraa@unican.es, ${ }^{4}$ suareza@unican.es
}

\begin{abstract}
An analysis of self-injection locked oscillators using a slow-wave structure for phase-noise reduction is presented. This structure is the key component of a feedback network, added to an existing oscillator and providing a stable self-injection locking signal. The unit cell of the slow-wave structure is based on a recently proposed configuration, made up of an open-ended stub and a Schiffman section. A tuning capacitor is introduced as an additional parameter, enabling an adjustment of the structure response at the desired oscillation frequency. The circuit solutions are analyzed by means of a semi-analytical formulation that incorporates the results of an electromagnetic simulation of the structure. The formulation enables a prediction of multivalued parameter regions, inherent to the long delay, which are more controllable than in the case of continuous transmission lines. An analytical derivation of the phase-noise spectral density is presented, which relates the phase-noise reduction with respect to the original freerunning oscillator to the group delay of the self-injection network. The analysis and synthesis method has been applied to an oscillator at $2.75 \mathrm{GHz}$.

Keywords-Oscillator, phase noise, stability.
\end{abstract}

\section{INTRODUCTION}

Phase noise is an undesired characteristic of oscillator circuits, which degrades their spectral purity and can induce demodulation errors. The possibility to reduce the phase noise of an existing oscillator through self-injection locking with long transmission lines has been demonstrated in several previous works [1]-[3]. However, this is generally inconvenient [3], since the overall system becomes bulky due to the long lengths required to achieve a significant phase-noise improvement. As shown here, the problem can be circumvented with slow-wave structures [4]-[5] implemented on microstrip line. Actually, oscillators based on slow-wave resonators with excellent performance have been demonstrated in the literature [6]-[7]. Here the slow-wave structure is included in the external feedback loop of an existing oscillator, in order to reduce its phase noise.

A complete analytical model of the self-injected oscillator is presented, which generalizes the one in [3], derived for a continuous transmission line, to the more complex case of slowwave structures. In particular, a recently proposed configuration, with a unit cell made up of an open-ended stub and a Schiffman section, is considered. A tuning capacitor is introduced as an additional parameter to adjust the structure response at the desired oscillation frequency.

Because the external feedback loop should not significantly modify the free-running operation point in terms of amplitude and frequency, the analysis will be based on a Taylor-series ex- pansion of the oscillator admittance function about the free-running solution of the standalone oscillator. The problem of coexistence of solutions in some parameter intervals, demonstrated in [3] and inherent to the long delay, will be shown to be less critical when using slow-wave structures. An analytical derivation of the phase-noise spectral density will be presented, relating the phase-noise reduction with respect to the original free-running oscillator to the group delay of the self-injection network. The analysis and synthesis method will be applied to an oscillator at $2.75 \mathrm{GHz}$, obtaining a phase-noise reduction of more than $13 \mathrm{~dB}$.

\section{Solution Curves When Using a Slow-WAVE Structure}

\section{A. Slow-wave structure}

The slow-wave factor $S_{W}$ of a given structure is the ratio between the wavelength in free space $\left(\lambda_{o}\right)$ and the wavelength when the signal propagates through the structure $\left(\lambda_{s}\right)$. Initially the case of a matched lumped-element transmission line, having an inductance $L_{c}$ and capacitance $C_{c}$ per unit cell of length $l_{c}$, is considered. The slow-wave factor and group delay are [4]:

$$
S_{W}=\frac{c}{l_{c} 2 \pi f} \arccos \left(1-\frac{2 f^{2}}{f_{c}^{2}}\right), \tau_{g}=\left(\pi f_{c} \sqrt{1-\frac{f^{2}}{f_{c}^{2}}}\right)^{-1}
$$

where $f_{c}$ is the Bragg frequency, given by: $f_{c}=1 /\left(\pi \sqrt{L_{c} C_{c}}\right)$. As gathered from (1), to increase the slow-wave factor, $l_{c}$ must be small and $L_{c}$ and $C_{c}$ must be large. The slow-wave structure will be implemented on microstrip, using the configuration proposed [5]. Its unit cell consists of a Schiffman-section [8], mainly contributing to the inductor, and an open-circuited stub, basically implementing the capacitor [Fig. 1(a)]. The inductance and capacitance per unit cell are [5]:

$$
\begin{aligned}
& L_{c} \cong \sqrt{Z_{o} Z_{e}} \beta_{f} 2 W / \omega \\
& C_{c} \cong \beta_{f} 2 W /\left(\sqrt{Z_{o} Z_{e}} \omega\right)+\beta_{b} W /\left(\omega Z_{b}\right)
\end{aligned}
$$

where $\omega=2 \pi f, Z_{o}$ and $Z_{e}$ are the odd and even mode impedances of the parallel coupled lines [5], $W$ is the transversal length, $\beta_{f}$ is the propagation constant of the Schiffman section and $\beta_{b}$ and $Z_{b}$ are the propagation constant and characteristic impedance of the line with the width $l_{b}$. 
The slow-wave structure will be applied to an oscillator at 2.75 GHz. To best fit the response of the structure to the oscillator frequency, a tuning capacitor is added in parallel to one of the middle cells [Fig. 1(b)]. From the ideal expressions in (1), with the unit cell values $L_{c}=1.89 \mathrm{nH}$ and $C_{c}=5 \mathrm{pF}$ and twelve cells, one should achieve a group delay of more than $2 \mathrm{~ns}$. The Bragg frequency is $3.27 \mathrm{GHz}$. Fig. 2 presents the comparison between the simulated and measured group delay of the structure in Fig. 1(b), when $W=9.4 \mathrm{~mm}$. The simulated group delay is obtained from an electromagnetic analysis of the slow-wave structure. Two different values of the tuning capacitor have been considered. The length of the structure is $36.2 \mathrm{~mm}$.

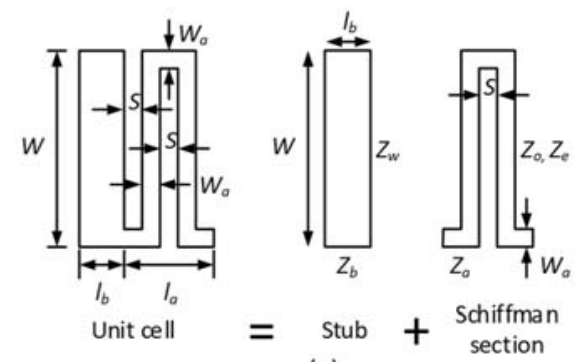

(a)

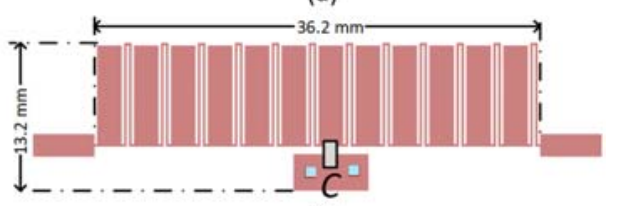

(b)

Fig. 1. Slow-wave structure consisting of Schiffman section and a stub. (a) Unit cell. (b) Complete structure, including a tuning capacitor $C$.

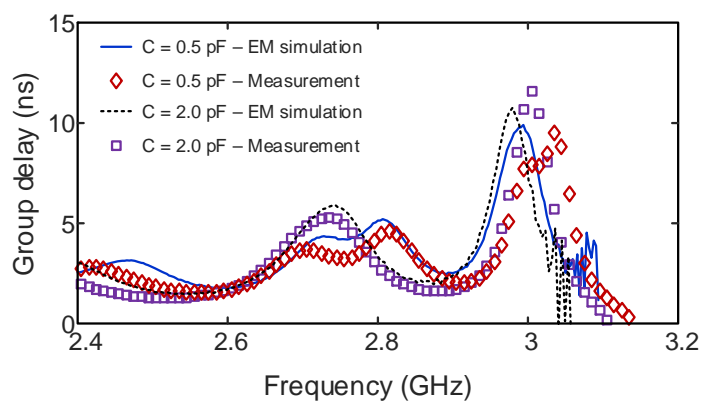

Fig. 2. Comparison between the simulated and measured group delay of the structure in Fig. 1(b), when $W=9.4 \mathrm{~mm}$, for two different $C$ values.

\section{B. Solution curves}

Initially, self-injection locking through the output port [1][2] will be considered, as shown in Fig. 3(a). This will allow an oscillator description in terms of its total admittance function $Y$ at the output node, constituting the only observation node [Fig. $3(\mathrm{~b})]$. The total admittance function is $Y(V, \omega)$, where $V$ and $\omega$ are the oscillation amplitude and frequency. In free-running conditions, that is, when the oscillator is terminated in $Y_{o}=1 / 50=0.02 \Omega^{-1}$, it fulfils: $Y\left(V_{o}, \omega_{o}\right)=0$. Now, the output $50 \Omega$ load will be replaced with the self-injection configuration in Fig. 3(a), made up of a circulator, a slow-wave structure and an attenuator. The equivalent input admittance of this configuration is $Y_{L}(\omega)$. In these conditions, the new steady-state equation at the fundamental frequency is:

$$
Y(V, \omega)-Y_{o}+Y_{L}(\omega)=Y(V, \omega)-Y_{o}+Y_{o} \frac{1-\Gamma_{L}(\omega)}{1+\Gamma_{L}(\omega)}=0
$$

where $\Gamma_{L}(\omega)=\rho(\omega) e^{j \phi(\omega)}$ is the reflection coefficient. The self-injection network must not significantly alter the free-running amplitude and frequency, so one can perform a Taylorseries expansion of $Y(V, \omega)$ about $V_{o}, \omega_{o}$. This provides:

$$
E=Y_{V}\left(V-V_{o}\right)+Y_{\omega}\left(\omega-\omega_{o}\right)-Y_{o}+Y_{L}(\omega)=0
$$

where $E$ is the error function and $Y_{V}$ and $Y_{\omega}$ are the derivatives of the admittance function, extracted from harmonic balance with the aid of an auxiliary generator [3]. System (3) particularizes to the one in [3] in the idealized case of a non-dispersive delay line. In that case, $\rho$ is constant and $\phi=\omega T$. When using, as done here, a slow-wave structure, there are no closed-form expressions for $\rho(\omega)$ and $\phi(\omega)$. The admittance $Y_{L}(\omega)$ is evaluated through an electromagnetic simulation of the slow-wave structure, as done in subsection $A$. Then the function is imported by in-house software, where equation (4) is solved by detecting the zeroes of the following function:

$$
\omega-\omega_{o}+\left(Y_{V}^{r} Y_{L}^{i}(\omega)+Y_{V}^{i} Y_{o}-Y_{V}^{i} Y_{L}^{r}(\omega)\right) /\left(Y_{V}^{r} Y_{\omega}^{i}-Y_{V}^{i} Y_{\omega}^{r}\right)=0
$$

where the superscripts $r$ and $i$ indicate real and imaginary parts.

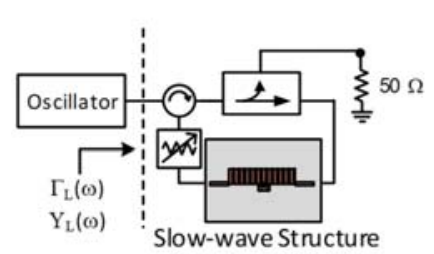

(a)

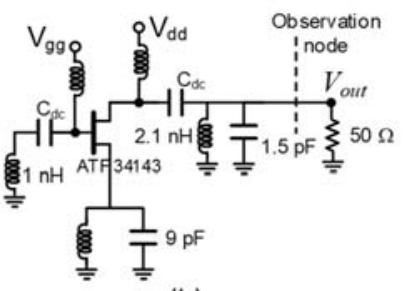

(b)
Fig. 3. Self-injection configuration. (a) Block diagram made up of a circulator, a slow-wave structure and an attenuator. (b) Oscillator circuit.

The analysis method has been applied to an oscillator based on PHEMT transistor ATF34143 operating at $2.75 \mathrm{GHz}$ [Fig. 3(b)]. Two sensitive parameters of the self-injected configuration will be the transversal length $W$ and the capacitor $C$. For a global evaluation of the performance, the length $W$ has been swept in the interval $9 \mathrm{~mm}$ to $11.5 \mathrm{~mm}$, for two values of the tuning capacitor $C$. The results are shown in Fig. 4(a), for $C=2 \mathrm{pF}$, and Fig. 4(b), for $C=1 \mathrm{pF}$. In the two cases, there are both single and multi-valued sections. As shown in Fig. 4(a) and (b), the location of the multivalued regions can be modified by changing the tuning capacitor.

To understand the causes of these multi-valued sections, one should analyze the impact of the group delay. This is better done by applying a Taylor series expansion to $Y_{L}(\omega)$ about 
$\Gamma_{L}=0$, which provides $Y_{L}(\omega) \cong Y_{o}\left[1-2 \Gamma_{L}(\omega)\right]$. This expression will be replaced into (4). At the turning points of the solution curves, the Jacobian matrix of the complex equation (4), calculated with respect to $V$ and $\omega$, becomes singular. This Jacobian matrix is:

$$
[J E]=\left[\begin{array}{cc}
Y_{V}^{r} & Y_{\omega}^{r}-2 Y_{o} \frac{\partial \rho}{\partial \omega} \cos \phi-2 Y_{o} \tau_{g} \rho \sin \phi \\
Y_{V}^{i} & Y_{\omega}^{i}-2 Y_{o} \frac{\partial \rho}{\partial \omega} \sin \phi+2 Y_{o} \tau_{g} \rho \cos \phi
\end{array}\right]
$$

where $-\partial \phi / \partial \omega=\tau_{g}(\omega)$ is the group delay associated with the input reflection coefficient. The determinant of (6) is:

$\operatorname{det}(\omega)=$

$\operatorname{det}_{o}+2 Y_{o}\left[\left(\tau_{g} \rho Y_{V}^{r}+\frac{\partial \rho}{\partial \omega} Y_{V}^{i}\right) \cos \phi+\left(\tau_{g} \rho Y_{V}^{i}-\frac{\partial \rho}{\partial \omega} Y_{V}^{r}\right) \sin \phi\right]^{(7)}$

where $\operatorname{det}_{o}=Y_{V}^{r} Y_{\omega}^{i}-Y_{V}^{i} Y_{\omega}^{r}$ is the determinant of the Jacobian matrix associated with the admittance function of the standalone free-running oscillator. The determinant det $_{o}$ does not depend on the frequency $\omega$ resulting from self-injection effects in system (3), unlike the situation with $\tau_{g}(\omega), \rho(\omega)$ and $\phi(\omega)$. According to [9], if the standalone oscillator is stable, one should have $\operatorname{det}_{\mathrm{o}}>0$, so the possible zero values will depend on the second term. For sufficiently large $\tau_{g} \rho$ and $\partial \rho / \partial \omega$, the sign of (7) will be sensitive to $\phi(\omega)$. Fig. 4(c) shows the variations of $\operatorname{det}(\omega)$ in (7). The turning points agree with the changes of sign in $\operatorname{det}(\omega)$. The sign of the dominant real pole of the self-injected oscillator is the opposite of that of $\operatorname{det}(\omega)$ [10]. Under a high attenuation magnitude, it is unlikely to get other dominant poles, so the stable sections should correspond to $\operatorname{det}(\omega)>0$. Nevertheless, it is advisable to verify this through a circuit-level stability analysis.

\section{PhASE-NoISE REDUCTION}

In order to get insight into the mechanism for phase-noise reduction, noise perturbations will be introduced in equation (3) , in the form of an equivalent noise current source $I_{N}(t)$, calculated by fitting the standalone free-running oscillator spectrum, as shown in [10]. This equivalent noise current will include the upconversion of flicker noise, modelled as proposed in [9]. The perturbed system is obtained by performing a Taylor-series expansion about the steady-state solution of (3):

$$
\begin{aligned}
& Y_{V} \delta V(t)+ \\
& {\left[Y_{\omega}-2 Y_{o} \frac{\partial \rho}{\partial \omega} e^{j \phi}-j 2 Y_{o} \rho \frac{\partial \phi}{\partial \omega} e^{j \phi}\right]\left[-j \frac{\delta \dot{V}(t)}{V_{o}}+\delta \dot{\phi}(t)\right]=\frac{I_{N}(t)}{V_{o}}}
\end{aligned}
$$

Neglecting also the time derivative of the amplitude increment $\delta \dot{V}(t)$, splitting (8) into real and imaginary parts and solving for $\delta \dot{\phi}(t)$, one obtains:

$\delta \dot{\phi}(t)=$

$\frac{Y_{V}^{r} I_{N}^{i}(t)-Y_{V}^{i} I_{N}^{r}(t)}{\operatorname{det}_{o}+2 Y_{o}\left[\left(\tau_{g} \rho Y_{V}^{r}+\frac{\partial \rho}{\partial \omega} Y_{V}^{i}\right) \cos \phi+\left(\tau_{g} \rho Y_{V}^{i}-\frac{\partial \rho}{\partial \omega} Y_{V}^{r}\right) \sin \phi\right]}$

Applying the Fourier transform, one obtains the following expression for the phase-noise spectral density:

$$
\begin{aligned}
& |\delta \phi(\Omega)|^{2}= \\
& \frac{\left|Y_{V}\right|^{2}(2 N+K / \Omega)}{\Omega^{2}\left\{d e t_{o}+2 Y_{o}\left[\left(\tau_{g} \rho Y_{V}^{r}+\frac{\partial \rho}{\partial \omega} Y_{V}^{i}\right) \cos \phi+\left(\tau_{g} \rho Y_{V}^{i}-\frac{\partial \rho}{\partial \omega} Y_{V}^{r}\right) \sin \phi\right]\right\}^{2}}
\end{aligned}
$$

where $N$ is the spectral density of the equivalent current source and $K$ accounts for the flicker noise coefficient and its upconversion effects. In the original free-running oscillator $\rho$ and $\partial \rho / \partial \omega$ are zero, so one can easily derive the phase-noise reduction due to the self-injection effects. This is approximately independent of the offset frequency $\Omega$, and given by:

$$
\begin{aligned}
& \Delta S(d B)=-20 \log 10\left(\operatorname{det}_{o}\right)+ \\
& 20 \log 10\left\{\operatorname{det}_{o}+2 Y_{o}\left[\left(\tau_{g} \rho Y_{V}^{r}+\frac{\partial \rho}{\partial \omega} Y_{V}^{i}\right) \cos \phi+\left(\tau_{g} \rho Y_{V}^{i}-\frac{\partial \rho}{\partial \omega} Y_{V}^{r}\right) \sin \phi\right]\right\}
\end{aligned}
$$

The phase-noise reduction $\Delta S(d B)$ potentially increases with the group delay $\tau_{g}$, which also depends on $\rho$ and $\phi$.
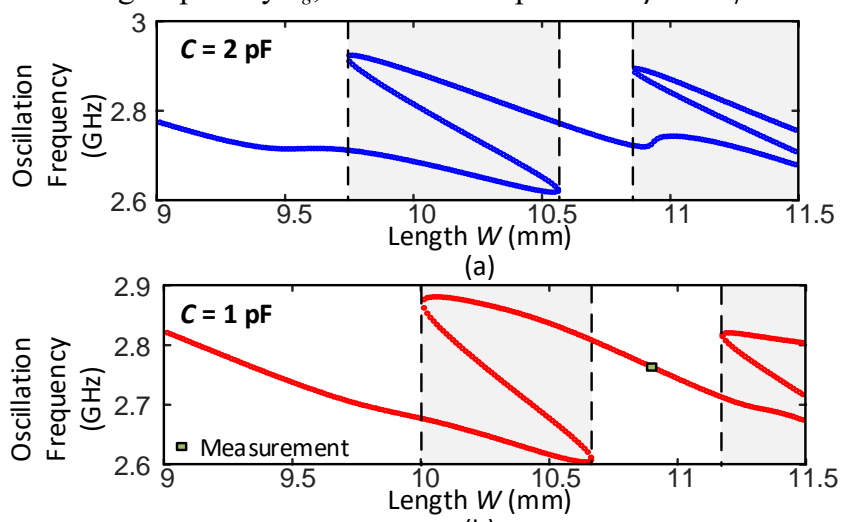

(b)

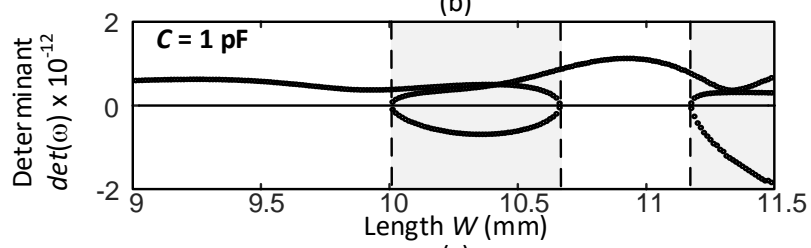

(c)

Fig. 4. Variation of the oscillation frequency versus the length $W$, swept in the interval $9 \mathrm{~mm}$ to $11.5 \mathrm{~mm}$, for two values of the tuning capacitor $C$. (a) $C=2$ $\mathrm{pF}$ (b) $C=1 \mathrm{pF}$. (c) Variations of the determinant $\operatorname{det}(\omega)$ for $C=1 \mathrm{pF}$. 
Fig. 5(a) presents the phase noise variation versus $W$, in the case of $C=1 \mathrm{pF}$, at the constant offset frequency $100 \mathrm{kHz}$. Note that, when neglecting $\delta \dot{V}(t)$ in (8), the phase noise tends to infinite at the turning points. At $100 \mathrm{kHz}$, the standalone oscillator exhibits a phase noise of $-85 \mathrm{dBc} / \mathrm{Hz}$. The chosen operation point corresponds to $W=10.9 \mathrm{~mm}$, in a single-valued region of the solution curve. The measured result, with $\Delta S=13 \mathrm{~dB}$, is also shown. Fig. 5(b) presents phase-noise variation versus the attenuation in the self-injection loop, with measurements superimposed. As can be seen, the phase noise reduction increases when reducing the attenuation. For too low attenuation, the curve becomes multi-valued. Fig. 6 presents a comparison of the phase-noise spectrum of the original free-running oscillator with that obtained with the self-injection configuration. Results obtained through the analytical formulation and through the conversion-matrix approach are validated with the experimental measurements. Note that, with a continuous transmission line, for $\operatorname{det}_{o}=2.32 \cdot 10^{-13} \Omega^{-1} \mathrm{~s} / \mathrm{V}$ and a similar $\Delta S$, a length of about $1.3 \mathrm{~m}$ would be required.

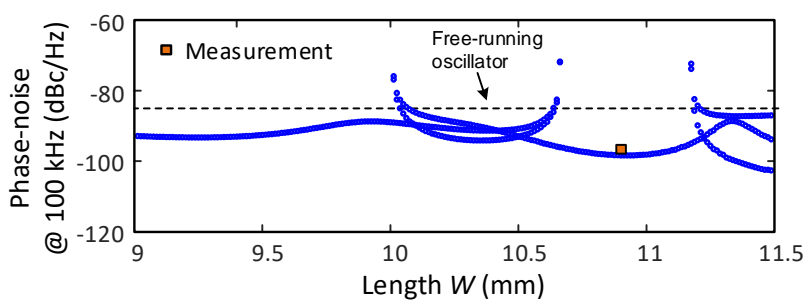

(a)

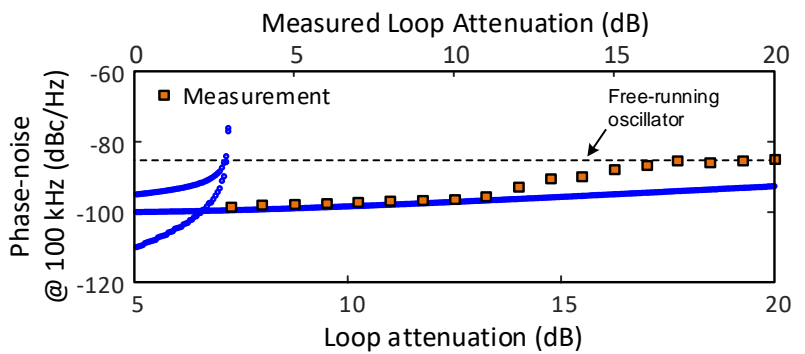

(b)

Fig. 5. Phase noise with a slow-wave structure in Fig. 1. (a) Versus $W$, in the case of $C=1 \mathrm{pF}$. The chosen operation point corresponds to $W=10.9 \mathrm{~mm}$. (b) Versus the attenuation in the loop.

Finally, a measurement of the phase-noise spectrum when introducing the slow-wave structure in an external feedback network, connected between the oscillator output and an auxiliary input port, is presented in Fig. 7. A reduction of $16 \mathrm{~dB}$ with respect to the free-running value is achieved.

\section{CONCLUSION}

The use of a slow-wave structure for the phase-noise reduction of an existing oscillator through self-injection locking has been presented. An expression relating the phase-noise spectral density to the group delay has been derived. Using a slow-wave structure based on a stub and Schiffman section, a phase-noise reduction of $13 \mathrm{~dB}$ has been achieved in a HEMT-based oscillator at $2.75 \mathrm{GHz}$.

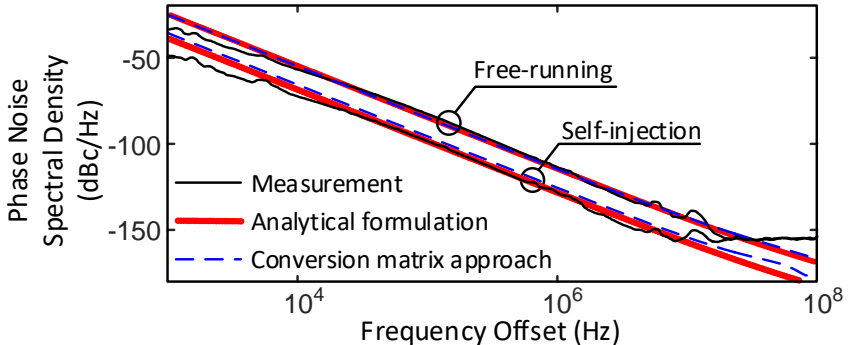

Fig. 6. Comparison of the phase-noise spectrum of the original free-running oscillator with that obtained with the self-injection configuration.

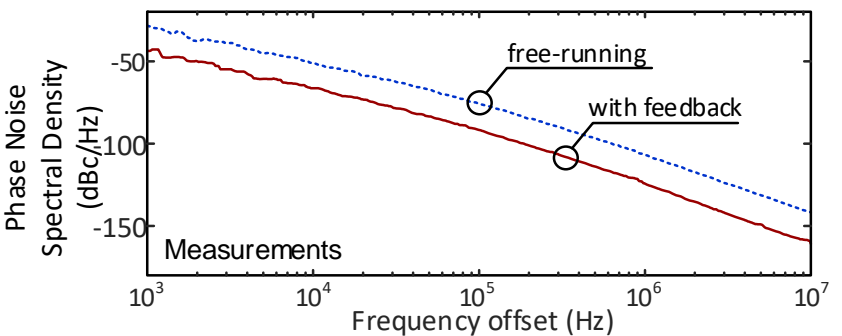

Fig. 7. Measurement of the phase-noise reduction when introducing the slowwave structure in an external feedback network, connected between the oscillator output and an auxiliary input port.

\section{ACKNOWLEDGMENT}

This work was supported by the Spanish Ministry of Science, Innovation and Universities and the European Regional Development Fund (ERDF/FEDER) under the research project TEC2017-88242-C3-1-R.

\section{REFERENCES}

[1] H.-C. Chang, "Stability analysis of self-injection-locked oscillators," IEEE Trans. Microw. Theory Techn., vol. 51, no. 9, pp. 1989-1993, Sept. 2003.

[2] H.-C. Chang, "Phase noise in self-injection-locked oscillators - theory and experiment," IEEE Trans. Microw. Theory Techn., vol. 51, no. 9, pp. 19941999, Sept. 2003.

[3] A. Suárez, F. Ramírez, "Analysis of Stabilization Circuits for Phase-Noise Reduction in Microwave Oscillators," IEEE Trans. Microw. Theory Techn., vol. 53, no. 9, pp. $2743-2751$, Sep. 2005.

[4] C. Zhou, H. Y. D. Yang, "Design Considerations of Miniaturized Least Dispersive Periodic Slow-Wave Structures," IEEE Trans. Microw. Theory Techn., vol. 56, no. 2, pp. 467-474, Feb. 2008.

[5] W.S. Chang, C.Y. Chang, "A high slow-wave factor microstrip structure with simple design formulas and its application to microwave circuit design," IEEE Trans. Microw. Theory Techn., vol. 60, no. 11, pp. 3376 3383, Nov. 2012.

[6] A. K. Poddar, U. L. Rohde, "Slow Wave Resonator based tunable oscillators," IEEE Int. Frequency Control and Europ. Frequency and Time Forum (FCS) Proc., San Francisco, CA, USA, 2011, pp. 1-10.

[7] A. K. Poddar, U. L. Rohde, "Slow-Wave Evanescent-Mode coupled resonator oscillator ciruits," IEEE Int. Frequency Control Symp. Proc., Baltimore, MD, 2012, pp. 1-7.

[8] B. Schiffman, "A new class of broad-band microwave 90-degree phase shifters," IRE Trans. Microw. Theory Techn., vol. 6, no. 2, pp. 232 -237, Apr. 1958.

[9] K. Kurokawa, "Some basic characteristics of broadband negative resistance oscillators", Bell Syst. Tech. J., vol. 48, pp. 1937-1955, July-Aug. 1969.

[10] F. Ramírez, M. Pontón, S. Sancho, A. Suárez, "Phase-Noise Analysis of Injection-Locked Oscillators and Analog Frequency Dividers", IEEE Trans. Microw. Theory Techn., vol. 56, no.2, pp. 393-407, 2008. 